\title{
Profiling Modulation of Interleukin-10 Immunosuppression Using Antibody Arrays
}

R\&D Systems, Inc., 614 McKinley Place NE, Minneapolis, MN, 55413

\section{Introduction}

The anti-inflammatory cytokine Interleukin-10 (IL-10) is known to be necessary for down regulating proinflammatory responses toward pathogens. IL-10 inhibits the production of many cytokines downstream of its receptor via the Jak1/Tyk2-STAT3 signaling pathway. The small molecule Tofacitinib, also called CP 690550, is a selective inhibitor of the Jak family of cytoplasmic tyrosine kinases. It has been shown to potently inhibit both Jak3- and Jak1-dependent STAT activation. Mouse microglial BV-2 cells are an established in vitro model for neuronal inflammation and have been reported to respond to IL-10 immunosuppression in a manner similar to primary mouse microglial cells. We used this model to examine the effects of Tofacitinib on the immunosuppression of proinflammatory cytokine secretion and the activation of the Jak/STAT signaling pathway by IL-10.

\section{Materials and Methods}

\section{Cell Culture and Treatments}

Immortalized mouse microglia BV-2 cells were cultured using DMEM (high glucose) supplemented with 5\% FBS containing 100 units $/ \mathrm{mL}$ penicillin and $100 \mu \mathrm{g} / \mathrm{mL}$ streptomycin, and maintained in a 5\% CO2 incubator at $37^{\circ} \mathrm{C}$. BV-2 cells remained untreated or were treated for one hour with $50 \mathrm{ng} / \mathrm{mL}$ Recombinant Mouse IL-10 (R\&D Systems, Catalog \# 417-ML) followed by $0.5 \mathrm{ng} / \mathrm{mL}$ Recombinant Mouse IFN- $\gamma$ (R\&D Systems, Catalog \# 485-MI) and $100 \mathrm{ng} / \mathrm{mL}$ E. coli Lipopolysaccharide type 0127:B8 (Sigma, Catalog \# L4516) for different durations. To investigate the effects of small molecule inhibitors, BV-2 cells were treated for one hour with DMSO carrier, $0.2 \mu \mathrm{M}$ Tofacitinib (Tocris, Catalog \# 4556), or 0.2
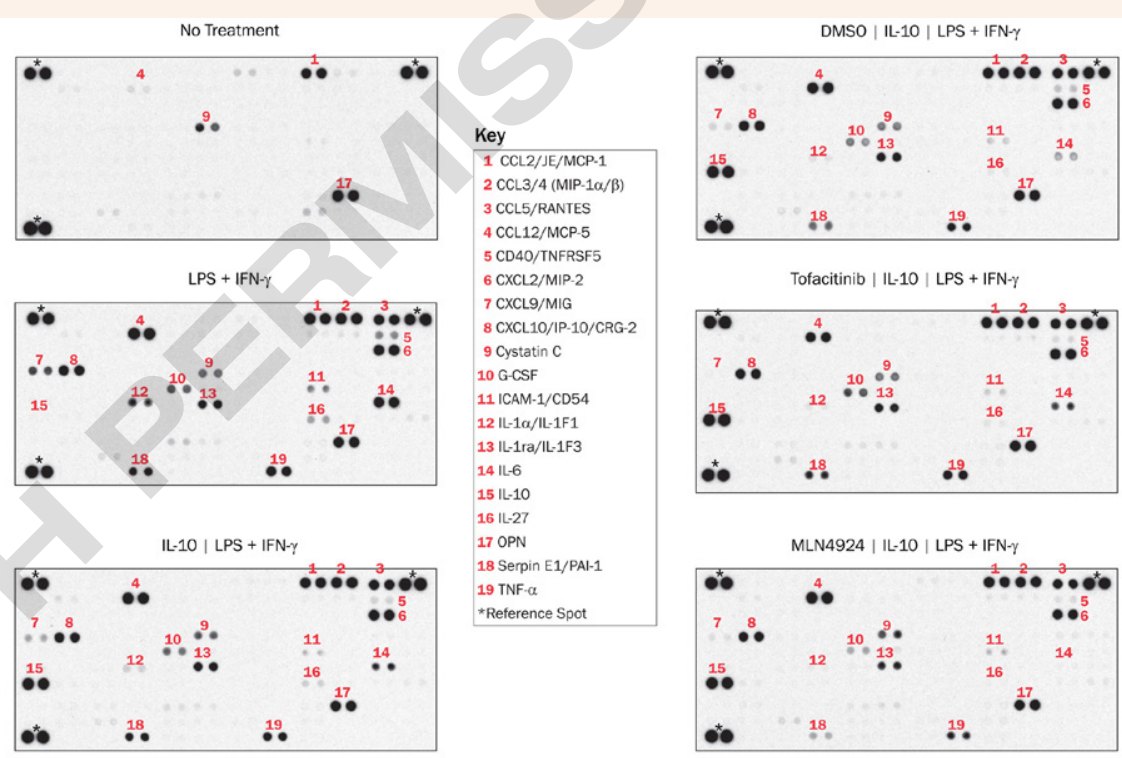
HM MLN4924 (R\&D Systems, Catalog \# I-502) prior to treatment with IL-10 and LPS $+\mathrm{IFN}-\gamma$.

Proteome Profiler ${ }^{\mathrm{TM}}$ Antibody Array Analysis

Cell culture supernates from BV-2 cells were analyzed using the Proteome Profiler ${ }^{\mathrm{TM}}$ Mouse XL Cytokine Array (R\&D Systems, Catalog \# ARY028).

\section{Summary}

Proinflammatory cytokines are secreted by mouse microglia BV-2 cells following activation of TLR4 by its agonist LPS. Pretreatment with IL-10, a potent anti-inflammatory cytokine, attenuates IFN- $\gamma$ and LPS-induced inflammatory cytokine secretion by BV-2 cells. The expression levels of CD40/TNFRSF5, CXCL9/MIG, G-CSF, ICAM-1/CD54, IL-1 $\alpha /$ IL-1F1, IL-6, IL-27, and TNF- $\alpha$ appeared to be attenuated. Tofacitinib, a selective Jak inhibitor, modulates microglia cytokine production induced by LPS and IFN- $\gamma$.

To learn more, please visit: rndsystems.com/Immunoassays.

BioTechniques 60:205 (April 2016) doi 10.2144/000114406 